\title{
Uso de una herramienta didáctica para la práctica de la entonación en hablantes no nativos de español
}

\author{
Pilar Oplustil - Pontificia Universidad Católica de Chile \\ psoplust@uc.cl \\ Gloria Toledo - Pontificia Universidad Católica de Chile \\ gtoledo@uc.cl
}

Rebut / Received: 21-9-17

Acceptat / Accepted: 20-3-18

Resum. Ús d'una eina didàctica per a la pràctica de l'entonació en parlants no nadius d'espanyol.
Aquest estudi parteix de la caracterització de l'entonació de les peticions i les ordres en l'espanyol de
Xile, amb l'objectiu de crear una eina computacional per a la didàctica de l'espanyol com a llengua
estrangera (ELE) i amb la finalitat de posar a prova aquesta eina entre 10 aprenents d'ELE. La
caracterització realitzada entre parlants xilens revela diferències entre l'entonació d'homes i dones pel
que fa als actes de parla abordats, i l'aplicació de l'eina entre els aprenents d'ELE posa de manifest la
major dificultat que tant els homes com les dones experimenten per imitar el patró entonatiu de les
ordres. D'altra banda, aquest estudi demostra els avantatges de l'eina, ja que el 100\% dels aprenents
va millorar la seva producció després d'haver-servit d'aquest recurs didàctic.

Paraules clau: espanyol com a llengua estrangera, entonació, eina didàctica, espanyol de Xile, fonètica i fonologia.

Abstract. A didactic tool for non-native Spanish speakers to practice intonation. This study starts from the characterization of intonation of requests and orders in Chilean Spanish in order to create a computational teaching tool for Spanish as a foreign language (SFL) and to test this tool among 10 SFL learners. The characterization of intonation among Chilean speakers reveals differences between men and women for the speech acts which are the object of the present study. The application of the tool among SFL learners shows a greater difficulty in imitating the intonational pattern of orders for both men and women. Finally this study shows the effectiveness of the teaching tool, since $100 \%$ of the learners improved their production after using this didactic resource. 
Keywords: Spanish as second language, intonation, teaching tool, Chilean Spanish, Phonetic and phonology

\section{Introducción}

La enseñanza de aspectos fonético-fonológicos es una necesidad reconocida en el ámbito del espańol como lengua extranjera (ELE), sin embargo, es perceptible una dificultad general entre los profesores del área para enseńar dicho componente. Esta situación, plantea la necesidad de realizar más estudios sobre temas de entonación en ELE; de lograr una mejor preparación para la didáctica de la entonación; y de desplazar el foco predominante en el estudio de los segmentos hacia los elementos suprasegmentales como la entonación, la acentuación, el ritmo o la velocidad, entre otros (Cortés, 2002; Hidalgo y Cabedo, 2011-2012).

Gil (2007) señala la importancia de que los aprendientes de ELE adquieran una buena pronunciación y atribuye un especial énfasis a la entonación, debido a "los efectos que a menudo produce en la transmisión y la interpretación correcta de los significados por parte del hablante y del oyente" (Gil, 2007, p. 98). Por su parte, Lahoz (2007) señala que aprender primero la entonación puede facilitar la adquisición de los segmentos, tomando en cuenta que esta se adquiere primero en la lengua materna.

De acuerdo al Plan Curricular del Instituto Cervantes (Instituto Cervantes, 2006) el ítem de pronunciación en la enseñanza de ELE debe comprender tres aspectos: una base de articulación general, aspectos suprasegmentales y fonemas vocálicos y consonánticos. De acuerdo al nivel de lengua del aprendiente, el plan distingue tres fases correspondientes a los tres aspectos recién mencionados: (1) la fase de aproximación en los niveles A1 y A2, en que el aprendiente debe conocer la base de articulación general; (2) la fase de profundización, para los niveles B1 y B2, que comprende el dominio de los aspectos suprasegmentales y (3) la fase de perfeccionamiento, en los niveles C1 y C2, que implica el conocimiento de los fonemas vocálicos y consonánticos.

Al margen de las propiedades universales, que podrían facilitar la transferencia positiva de los aprendientes (Gil, 2007), la entonación del espańol posee aspectos propios que la diferencian de otras lenguas, en consecuencia, debiera enseñarse formalmente. Obsérvese como ejemplo, que el ascenso al final de la curva de entonación en preguntas - considerado un rasgo común a todas las lenguas - no sucede en el danés, el búlgaro, el ruso, el finés, el húngaro, el árabe occidental y el portugués de Brasil (Gil, 2007), de forma tal que entre los hablantes de todas esas lenguas, ese rasgo debe ser trabajado.

Tomando en cuenta todo lo anterior, este estudio aborda la práctica de la entonación en ELE, empleando un prototipo de herramienta didáctica computacional para la enseñanza de la entonación del español de Chile (Oplustil, 2016). La práctica con esta herramienta fue examinada con 10 aprendientes de niveles B1-B2. La elección de este segmento se vincula con el planteamiento del PCIC, de acuerdo al cual la identificación 
y producción de los patrones melódicos correspondientes a los distintos actos de habla, se deben enseñar en estos niveles. El uso sistemático de este tipo de programas ${ }^{1}$ facilita la práctica entonacional y produce una mejora en relación con la entonación de los hablantes nativos (Gil, 2007).

\section{La entonación en espańol}

La entonación se define como "el fenómeno lingüístico que constituyen las variaciones de tono relevantes en el discurso oral. Se trata del primer elemento cohesionador del habla, que además cumple funciones lingüísticas y expresivas en la comunicación oral” (Cantero, 2002, p. 15). Gracias a estas funciones, la entonación puede determinar cuándo un mismo enunciado es una petición o una orden, ya que, si bien el contexto situacional puede proporcionar pistas sobre la fuerza ilocutiva del acto de habla, la entonación es la que contribuye en mayor medida a su distinción.

La entonación es a la vez un fenómeno fonético y fonológico, de carácter suprasegmental. Como parte de la prosodia, la entonación consiste en la melodía de los enunciados. Como fenómeno fonológico, produce diferentes efectos lingüísticos, al comportarse como un sistema de patrones propios para cada lengua. En el caso de una lengua entonativa, como el español, la entonación comunica significados semánticos y pragmáticos (Prieto, 2003). Cuando los cambios de frecuencia fundamental $(\mathrm{f} 0)^{2}$ de la melodía pasan a ser estructurados y opositivos, se convierten en unidades fonológicas que portan información lingüística; la oposición entre un tonema ascendente y uno descendente genera distinciones entre enunciados interrogativos y declarativos, respectivamente.

El español posee algunas características generales en su entonación, entre ellas, la declinación general del rango de f0 en la entonación neutra, a medida que avanza el tiempo (Martínez y Fernández, 2007), en oposición a la entonación interrogativa absoluta, en que el rango suele aumentar hacia el final del enunciado. La alteración del rango de fo hacia el final del enunciado también se utiliza para dar énfasis o para indicar otros valores semánticos o pragmáticos específicos (Martínez y Fernández, 2007).

Navarro Tomás (1996) señala dos curvas prototípicas (Fig. 1) para los tipos de entonaciones mencionados, en las cuales distingue tres momentos de la curva melódica: (1) el tonema, que corresponde al último acento de la frase y al resto de las sílabas no acentuadas hasta el final del enunciado; (2) el inicio, que corresponde a las sílabas no acentuadas anteriores a la primera sílaba acentuada, y por último, (3) el cuerpo, que corresponde al conjunto de sílabas incluyendo la primera sílaba acentuada y las subsiguientes, hasta el inicio del tonema.

1. Para un resumen de los distintos programas para la entonación en español, v. Llisterri (2001).

2. F0 es la diferencia en frecuencia entre los armónicos que componen un sonido complejo. 


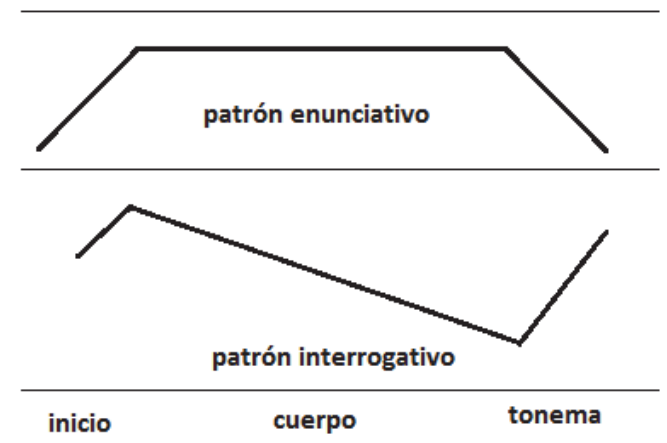

Figura i. CURVAS PROTOtípicas SEgún NaVARro Tomás (I996).

El español no tiende a la entonación plana, sino a una sucesión de movimientos tonales, en que se repiten ascensos y descensos (Martínez y Fernández, 2007). Tres tonemas se distinguen para el español: ascendente, descendente y suspensivo. De manera general, el tonema ascendente se utiliza para expresar ideas no concluidas o que esperan una respuesta (Gil, 2007). Este tonema se presenta en grupos enunciativos, enunciados declarativos insinuativos, enunciados interrogativos que genuinamente solicitan información como respuesta, preguntas retóricas y algunos enunciados de ruego o mandato. El tonema descendente se emplea para enunciados que indican conclusión o poseen una completitud de sentido (Gil, 2007). Entre estos, se encuentran las respuestas categóricas a preguntas, elementos internos de la enumeración, vocativos, preguntas de eco e imperativos y ruegos insistentes, entre otros. El tonema suspensivo se produce internamente en enunciados con dos grupos, por ejemplo, al intercalar un inciso o cuando un enunciado queda en suspenso (Gil, 2007).

Sobre las entonaciones en distinto tipo de enunciados, Garrido (2001) realiza una propuesta de modelización para las curvas melódicas en español, y además señala la necesidad de identificar el grado de vinculación que existe entre los patrones melódicos y los actos de habla. Destacamos los estudios de Navarro Tomás (1966), Hidalgo (2006a) y Cantero y Font (2007), sobre el español peninsular; el de Álvarez (2005), para el español de Argentina; y los de Orozco (2005), para el español de México.

En relación con el estudio de los valores de (des)cortesía de la entonación están los trabajos de Álvarez y Blondet (2003), sobre cortesía y prosodia en el español de Mérida; los de Hidalgo (2006b y 2007), que consideran la prosodia para analizar la cortesía en la conversación; los de Hidalgo (2009) y Culpeper (2011), sobre la expresión de descortesía mediante la prosodia; los de Hidalgo y Martínez (2017), que aportan una descripción del componente prosódico en la atenuación, precisando parámetros acústicos objetivables; y el de Orozco (2008), en que compara la producción de peticiones en estilo neutro o 
cortés, para presentar un patrón entonativo de dichos actos de habla, a partir de una prueba con hablantes nativos de Guadalajara, México.

Entre los autores que han abordado el tema en el español de Chile, se encuentran Roldán (2000) y Fuentes (2012). Roldán (2000) estudió las variaciones en patrones melódicos de atenuación de la fuerza ilocutiva de los actos de habla, mediante un corpus consistente en conversaciones televisivas y radiales. Entre sus conclusiones, señala que la entonación participa de la atenuación a través de características tales como prominencias o elevaciones de tono muy marcadas, un patrón melódico sinuoso en el cuerpo, juntura terminal predominantemente descendente y rango de entonación amplio (Roldán, 2000). Fuentes (2012), por su parte, realizó un análisis prosódico para peticiones y órdenes, a través del modelo de la teoría autosegmental $\mathrm{AM}^{3}$, empleando un corpus de pares de enunciados idénticos que, según un contexto dado al hablante, eran entonados como orden o como petición (Fuentes, 2012, p. 27). Su análisis consistió tanto en el aspecto prosódico de la duración, como en la frecuencia fundamental. En términos de frecuencia fundamental, los resultados señalan que, en cuanto al tonema, las órdenes poseen un descenso marcado, en cambio, la distribución entre tonema ascendente y descendente para las peticiones fue casi equivalente porcentualmente (Fuentes, 2012).

\section{Metodología}

\subsection{Procesamiento computacional de la curva melódica}

El primer paso que se dio en este estudio consistió en analizar y describir la entonación de un corpus de peticiones y órdenes del español de Chile, para obtener un modelo representativo del patrón entonativo de tales actos de habla (Oplustil, 2016) que luego pudieran imitar los aprendientes de ELE. Gracias al procesamiento computacional de la curva melódica se obtuvieron los patrones entonativos necesarios para construir la herramienta didáctica. Esta etapa permitió despejar algunas dudas, ya que los resultados no son concluyentes entre las descripciones relacionadas con la entonación de las órdenes y las peticiones del español, las cuales se han centrado principalmente en identificar un tonema característico. Por ejemplo, Roldán (2000) señala que un 80\% de las peticiones presentan un tonema con descenso, sin embargo, Fuentes (2012) muestra que la distribución de tonemas ascendente y descendente es casi equivalente. El modelo de procesamiento realizado en este estudio se propuso lograr una descripción general más consistente, que incluyera toda la curva entonativa y no solo el tonema o ciertos acentos de la curva. Buscamos que el procesamiento computacional ofreciera

3. El modelo AM entiende que el contorno entonativo de un enunciado es el producto de la interpolación fonética entre eventos tonales asociados con sílabas definidas. Básicamente, a nivel fonológico, los tonos pueden estar asociados con sílabas que tienen acento léxico, es decir acentos tonales, o con el final de ciertas frases. 
resultados compatibles con el funcionamiento de la herramienta didáctica, tanto en el reconocimiento de la emisión entrante, como en su evaluación. Los resultados de este procesamiento fueron analizados estadísticamente para extraer una caracterización de los actos de habla analizados, que luego serían utilizados para que la herramienta didáctica corrigiera las emisiones de los aprendientes de ELE.

Para realizar el procesamiento computacional se empleó el corpus de emisiones recopilado y descrito en detalle por Fuentes (2012). De ese corpus de 425 producciones, correspondientes a 20 informantes en total, se seleccionaron 22 enunciados iguales por hablante; 11 peticiones y 11 órdenes. Los enunciados eran los mismos para ambos actos de habla, de forma tal que se les entregó a los informantes un breve contexto que les permitió inferir la fuerza ilocutiva de la emisión, para adecuar su curva melódica. Del procesamiento automático de la entonación se obtuvieron tres características: secuencia de movimiento tonales de la frecuencia fundamental; características del rango en semitonos de la entonación y características de la duración de las emisiones.

Una vez obtenidos los datos que fijaron los parámetros de la herramienta didáctica para la entonación, esta fue probada con estudiantes de ELE en la Pontificia Universidad Católica de Chile, de niveles B1 y B2. Ocho de ellos eran hablantes nativos de inglés y otros dos eran japoneses. En dos instancias, separadas por una semana, los aprendientes usaron la herramienta y registraron su desempeño.

\subsection{Caracterización por grupo: patrones generales}

La Tabla 1 reúne los resultados obtenidos en el procesamiento computacional del corpus de órdenes y peticiones, configurando los valores que la herramienta didáctica tendrá como referencia. La secuencia tonal escogida responde a la combinación más frecuente de movimientos. A partir de estos resultados, se fundamentará el criterio de evaluación de la herramienta didáctica.

TABla i. Resumen DE LOS RESUltados

\begin{tabular}{|c|c|c|c|c|}
\hline Rasgo/Grupo & $\begin{array}{l}\text { Orden/ } \\
\text { Hombre }\end{array}$ & $\begin{array}{l}\text { Petición/ } \\
\text { Hombre }\end{array}$ & $\begin{array}{l}\text { Orden/ } \\
\text { Mujer }\end{array}$ & $\begin{array}{c}\text { Petición/ } \\
\text { Mujer }\end{array}$ \\
\hline $\begin{array}{c}\text { Duración (seg.) } \\
\text { Promedio }\end{array}$ & 0,8762 & 1,0606 & 0,9274 & 1,0254 \\
\hline Máximo & 1,21 & 1,37 & 1,19 & 1,28 \\
\hline Mínimo & 0,54 & 0,75 & 0,67 & 0,78 \\
\hline Secuencia tonal ${ }^{4}$ & 21113 & 31111 & 21133 & 33111 \\
\hline
\end{tabular}

4. La secuencia tonal es un patrón de 5 valores donde 1 corresponde a un movimiento plano, 2 ascendente y 3 descendente. Se llegó a una división de 5 segmentos de manera empírica (Oplustil, 2016). 


\begin{tabular}{|r|c|c|c|c|}
\hline \multicolumn{2}{|l|}{ Rango (st) } & & & \\
Promedio & 11,6858 & 10,3236 & 14,7055 & 13,3429 \\
\hline Máximo & 16,97 & 14,27 & 23,45 & 19,17 \\
\hline Mínimo & 6,40 & 6,37 & 5,97 & 7,51 \\
\hline
\end{tabular}

Los resultados del procesamiento computacional automático permitieron encontrar algunos rasgos distintivos entre la entonación de órdenes y peticiones en este corpus, como se resume en la Tabla 2.

TABla 2. Resumen de los RASGOS ENTONATIVOS RELEVANTES ENCONTRAdos PARA LAS PETICIONES Y ÓRDENES (OPLUSTIL, 20i6, P. 259)

\begin{tabular}{|c|c|c|}
\hline & Órdenes & Peticiones \\
\hline Hombres & $\begin{array}{l}\text { Posee un patrón predominante de forma } \\
\text { trapecio (enunciativo). El segundo patrón } \\
\text { predominante es una curva plana. Nunca } \\
\text { comienzan con descenso. Raramente } \\
\text { finalizan con ascenso. Predomina el final } \\
\text { con descenso. Suelen tener un rango en st } \\
\text { un poco más amplio que las peticiones. }\end{array}$ & $\begin{array}{l}\text { Posee un patrón predominante de } \\
\text { forma descenso-plana. El segundo } \\
\text { patrón predominante es una } \\
\text { curva plana con descenso. Suelen } \\
\text { durar más que las órdenes. Se } \\
\text { hace un mayor contraste en } \\
\text { duración con las órdenes. }\end{array}$ \\
\hline Mujeres & $\begin{array}{l}\text { Posee un patrón predominante de forma } \\
\text { trapecio (enunciativo). El segundo patrón } \\
\text { predominante es una curva plana con } \\
\text { descenso final. Muy pocas veces comienza } \\
\text { con descenso. Raramente finalizan } \\
\text { con ascenso. Predomina el final con } \\
\text { descenso. Suelen tener un rango en st } \\
\text { un poco más amplio que las peticiones. }\end{array}$ & $\begin{array}{l}\text { Posee un patrón predominante de } \\
\text { forma descenso-plana (con énfasis). } \\
\text { El segundo patrón predominante } \\
\text { es una curva plana con ascenso } \\
\text { final. Suelen durar más que las } \\
\text { órdenes. Se realizan a través } \\
\text { de numerosas configuraciones } \\
\text { de la curva melódica. }\end{array}$ \\
\hline
\end{tabular}

Como un resultado indirecto de este estudio, se encontraron diferencias entre la entonación de hombres y mujeres para los actos de habla abordados. Hidalgo (2002) señala que la diversidad sexolingüística, expresada mediante la entonación, puede responder a causas tales como patrones entonativos esterotípicamente identificados con hombres y mujeres, para establecer relaciones de dominación; o el empleo de diferentes estrategias comunicativas entre hombres y mujeres.

\subsection{Descripción de la herramienta didáctica}

Desde el punto de vista de la enseñanza de lenguas, el foco de la herramienta didáctica es desarrollar las habilidades comunicativas del usuario respecto a la entonación, y de manera indirecta, sus habilidades pragmáticas. De hecho, escogieron las peticiones y 
las órdenes por su importancia pragmática en términos de cortesía y las consecuencias socialmente negativas que puede traer a un aprendiente de ELE no manejar la entonación de estos actos de habla. A estos actos, la herramienta entrega un pequeńo marco comunicativo del cual se puede inferir la jerarquía de los hablantes y otros elementos contextuales como la relevancia de lo que se pide.

Puesto que la herramienta es un prototipo, sólo se diseñaron dos ejemplos para cada acto de habla. Para cada uno se grabó una emisión de referencia de un hablante hombre y de una hablante mujer; cuatro ejemplos en total. Los informantes oscilaban entre los 20 y 25 años, eran hablantes nativos del español de Chile y originarios de la ciudad de Santiago. Los ejemplos grabados son los siguientes: a) Petición. Contexto: Vas a tomar el Metro junto a un amigo, pero no te alcanza el dinero para el pasaje. Entonces, le dices: Préstame plata. b) Orden. Contexto: Tarde en la noche, tratas de dormir, pero el hijo de tu vecino hace una fiesta y tiene la música muy fuerte. Ya le has pedido numerosas veces que baje el volumen y finalmente le ordenas: Baja la música.

Como se puede ver, el usuario debe inferir del ejemplo cuáles son las condiciones de uso de la orden: la edad del interlocutor, la jerarquía y un contexto específico en que un hablante recurriría a este tipo de estrategia. Es importante destacar que cuando la herramienta didáctica analiza la emisión entrante para ver cómo fue realizada, la entonación la compara con los patrones generalizados obtenidos del procesamiento de la curva melódica. En otras palabras, el usuario no estará aprendiendo a hablar como el informante de los ejemplos, estará aprendiendo los rasgos promedio para este tipo de entonación. Con los ejemplos, el usuario dispone de emisiones que puede escuchar las veces que sean necesarias para imitar su entonación.

\subsection{Interfaz gráfica y modo de uso}

El prototipo se programa en la pantalla demo de Praat (Boersma y Weenick, 2013), la cual permite desarrollar de manera fácil una interfaz gráfica simple, que facilita el uso del programa. En primer lugar, se le pregunta al usuario si es hombre o mujer, para seleccionar los ejemplos correspondientes a su sexo (Fig. 2). Luego, el usuario podrá escoger entre practicar órdenes o peticiones (Fig. 3). Una vez que haya escogido, la pantalla desplegará textualmente el contexto de la situación. El usuario verá escrita la emisión que debe realizar y también podrá escucharla, mediante un archivo de audio que funciona como ejemplo (si le cuesta percibir el enunciado, tendrá el apoyo gráfico escrito) (Fig. 4). Luego, el usuario debe seguir los pasos que le permitirán grabar su emisión. Finalmente, le pedirá al programa que corrija la emisión realizada.

El botón Instrucciones para grabar (Fig. 4) informa brevemente cómo grabar con la ventana de Praat. 
Prototipo herramienta didáctica

Selecciona una alternativa

\section{Mujer}

Fig. 2. Selección del SEXo.

Tarde en la noche, tratas de dormir pero el hijo de tu vecino hace una fiesta y tiene la música muy fuerte. Ya le has pedido numerosas veces que baje el volumen y finalmente le ordenas:

'Baja la música'

Instrucciones para Grabar

Volver Escuchar ejemplo

Grabar

\section{Corregir}

Fig. 4. Contexto ejemplo.

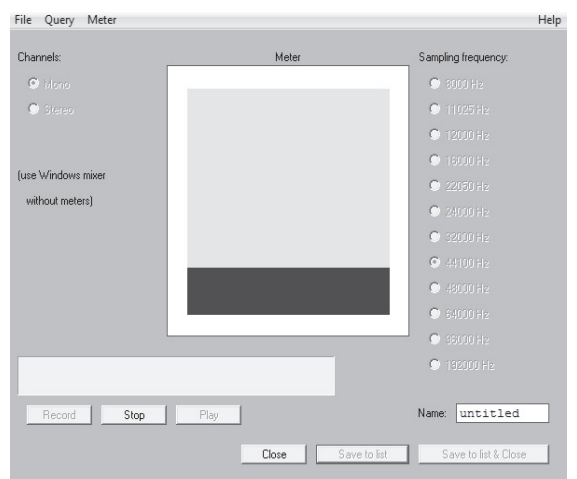

Fig. 5. Ventana de grabación de Praat.

Una vez que el usuario se ha grabado y ha guardado su emisión, se evalúa presionando el botón Corregir (Fig. 4). El usuario puede conocer el detalle de la evaluación presionando el botón Detalles (Fig. 6), que desplegará una ventana de información con los valores obtenidos, los valores esperados y el porcentaje de similitud para cada rasgo (Fig. 6). Finalmente, los tres porcentajes se promedian y se obtiene el porcentaje de similitud al centro de la pantalla 5 (Fig. 6). Adicionalmente, el usuario puede escuchar la emisión que grabó presionando Emisión (Fig. 6) y obtener una comparación gráfica de la curva melódica de su emisión y la emisión esperada, al presionar Gráfico (Fig. 6). La ventana de imágenes de Praat mostrará las curvas comparadas (Fig. 7); en rojo la entonación esperada y en verde la entonación del usuario. 


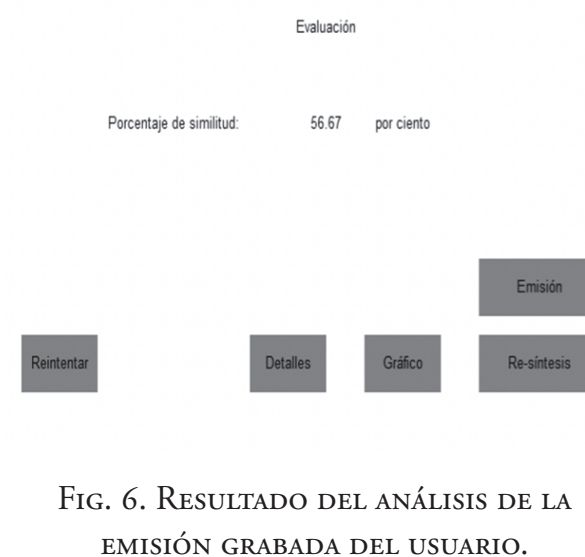

EMISIÓN GRABADA DEL USUARIO.

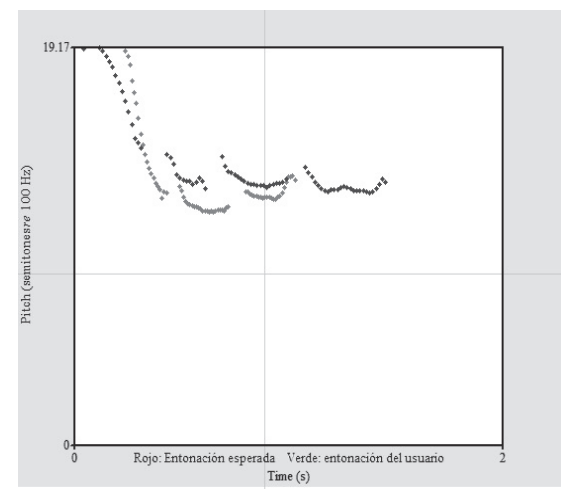

Fig. 7. GRÁfico QUe COMPARA LAS CURVAS ENTONACIONALES “CORRECTA" Y DEL USUARIO.

\section{Prueba del prototipo de herramienta didáctica con aprendientes de ELE}

Durante una primera sesión de recogida de datos para probar la herramienta, se les pidió a los aprendientes que grabaran su emisión sin escuchar el ejemplo, a objeto de contrastar su progreso con una segunda emisión, previo a la cual sí escucharían la entonación esperada. En promedio, el logro obtenido después de escuchar el ejemplo fue de un 22, 3\% más de similitud en la orden y un 24,6\% en la petición.

Para la petición, la mayoría de los participantes obtuvo un 100\% de logro en la segunda emisión. Tres de ellos no mostraron ningún progreso, dado que dos usuarios habían obtenido un $100 \%$ en la primera emisión y un tercero no logró superar el $83,33 \%$. Este caso que, obtuvo un $100 \%$ de similitud en la secuencia tonal, no logró imitar la velocidad y el rango en semitono (st) del ejemplo. Otro de los diez participantes subió un porcentaje muy bajo de 6,67\% en la segunda emisión de la petición, porque su logro en la primera ya había sido bastante alto. Aquellos usuarios que aumentaron en un $40 \%$ la similitud lograron un $100 \%$ de logro en las peticiones durante la segunda emisión. El mayor porcentaje de similitud en la emisión de la orden fue de 93,33\%, sin que ninguno de los aprendientes registrara una producción idéntica al ejemplo. En la emisión de las órdenes siempre se registró un porcentaje de correspondencia con la secuencia tonal inferior al porcentaje total de similitud logrado, lo cual indica una mayor dificultad para imitar los movimientos tonales, en relación con la velocidad y el rango en st. Sin embargo, en las peticiones se registraron cuatro casos en que el porcentaje de movimientos tonales correctos fue mayor que el porcentaje total de similitud, lo cual muestra una dificultad relacionada más con la velocidad y el rango en st.

En la segunda sesión de grabación, con una semana de desfase, la gran mayoría de los aprendientes mantuvo los puntajes de la segunda emisión en la primera sesión (después 
de escuchar el ejemplo). De los diez participantes, solo se registró un caso que en la segunda sesión tuvo una primera emisión con un porcentaje más alto que durante la primera sesión: 1,66\% más en la orden y un 16,67\% más en la petición.

En resumen, los hablantes no nativos que usaron la herramienta muestran más problemas en la emisión de la orden, y una mayor dificultad para imitar los movimientos tonales de esta. Ninguno de los participantes logró producir el movimiento ascendente con que comienza la orden. En la petición, por otra parte, se observó importantes logros; solo un estudiante de nacionalidad japonesa no logró producir un 100\% de similitud con la petición, ya que mantuvo problemas con la imitación de la velocidad y el rango en st.

Finalmente, en términos de usabilidad de la herramienta, se observó que los datos más comprensibles para los aprendientes eran el porcentaje de similitud entre la emisión del usuario y el de la lengua meta; los movimientos tonales obtenidos, en relación con la secuencia tonal esperada, y el porcentaje de secuencia tonal obtenido.

\subsection{Ventajas y limitaciones de la herramienta didáctica}

Dentro de las ventajas, podemos destacar que: (1) el programa no toma en cuenta los segmentos que emite el hablante, por lo tanto, aunque este no los domine aún a la perfección, puede ya entrenarse a nivel entonacional en la lengua meta; (2) el usuario recibe distintos modos de retroalimentación, desde gráficos que representan la entonación hasta una cuantificación de su desempeño; (3) la evaluación de la emisión del usuario/ aprendiente de español en Chile, está siendo evaluada objetivamente al ser comparada contra patrones obtenidos de un corpus de emisiones del espańol de Chile; (4) el prototipo es escalable y por lo tanto, teniendo conocimiento de programación en Praat, fonetistas y/u otros expertos pueden incluir nuevos ejemplos y/o contextos, o bien modificar los existentes.

Algunas desventajas son que la grabación puede tener problemas si hay ruido o si el usuario deja pasar mucho tiempo entre la activación de la grabación y el momento en que comienza a hablar. Ese segmento de tiempo puede ser mal editado por el algoritmo y entregar un análisis falso al determinar el primer movimiento tonal. Para solucionar este problema se puede entregar instrucciones más detalladas al usuario de cómo grabar, restringir el tiempo de grabación, o mejorar la primera segmentación que hace el algoritmo de la curva melódica.

En términos técnicos, la herramienta está usando técnicas básicas de síntesis y reconocimiento de voz a través de Praat, para llevar a cabo la evaluación de la emisión del alumno así como la resíntesis. El análisis de la emisión del alumno se podría llevar a cabo con sistemas como un vocoder, por ejemplo, Straight (Kawahara, Estill y Fujimura, 2001), entregando mayor rapidez y posibilidad de integración que Praat. El mismo permitiría resintetizar emisiones, modificando los valores de frecuencia fundamental y otros valores acústicos, para entregar una versión mejorada de la voz del alumno. 


\section{Conclusión}

Hemos mostrado el resultado de dos estudios relacionados con la creación y aplicación de una herramienta didáctica: uno para precisar las características entonativas de órdenes y peticiones en mujeres y hombres chilenos, y el uso de la herramienta por parte de hablantes no nativos de español, para determinar cómo los beneficia este recurso didáctico y cuáles son los rasgos que más les cuesta lograr para imitar la entonación nativa de mujeres y hombres hablantes del español de Chile.

A partir de ambos estudios se observó primeramente que la producción de órdenes entre hombres y mujeres comparte rasgos tales como los siguientes: predomina un patrón similar al enunciativo; nunca comienzan con un descenso tonal; predomina el final con descenso; y muestran un rango en st. más amplio que las peticiones. Respecto a las peticiones, hombres y mujeres comparten un patrón predominante de forma descensoplana, pero las mujeres agregan un énfasis. Entre hombres y mujeres, este acto de habla dura más que las órdenes y, entre mujeres, se realiza a través de variadas configuraciones de la curva melódica. En este punto sería muy interesante complementar, con los resultados obtenidos en Chile, los estudios de Hidalgo (2002) para determinar por qué las mujeres cuentan con más variedad de patrones que los hombres; cómo el contexto puede determinar la elección de uno u otro, y si es factible que los hombres aprovechen el rasgo de duración como un contraste enunciativo.

En cuanto al estudio entre hablantes no nativos, se pudo constatar una mayor dificultad para imitar el patrón entonativo de las órdenes, tanto en hombres como en mujeres. Al parecer, el rasgo más problemático de adquirir es la configuración de la curva melódica con movimiento inicial ascendente en las órdenes. Por otra parte, salvo un solo caso, el logro de similitud para las peticiones, tras la práctica con la herramienta, fue un éxito entre los aprendientes y, de manera general, el 100\% de los casos mejoró su producción tras escuchar el ejemplo que entrega este recurso. Queda pendiente optimizar la herramienta didáctica incorporando nuevos actos de habla, para estudiar el desempeńo de los hablantes no nativos y determinar nuevas dificultades en la entonación de estos.

\section{Referencias}

Álvarez, G. (2005). Contornos de entonación en el discurso de una docente: su relación con algunos aspectos pragmáticos. Onomázein, 11(1), 67-85.

Álvarez, A., y Blondet, M. A. (2003). Cortesía y prosodia: Un estudio de la frase cortés en el español de Mérida (Venezuela). En P. Martín Butragueño, Z. E. Herrera (Eds.), La tonía. Dimensiones fonéticas y fonológicas (pp. 319-330). México: El Colegio de México. 
Boersma, P., y Weenink, D. (2013). Praat: Doing phonetics by computer [Computer program]. Version 5.3.53. Recuperado de http://www.praat.org/

Cantero, F. J. (2002). Teoría y análisis de la entonación. Barcelona: Edicions Universitat de Barcelona.

Cantero, F. J., y Font, D. (2007). Entonación del español peninsular en habla espontánea: patrones melódicos y márgenes de dispersión. Moenia: Revista Lucense de Lingüistica y Literatura, 13, 69-92.

Cortés, M. (2002). Didáctica de la entonación: Una asignatura pendiente. Didáctica (Lengua y Literatura), 14, 65-75.

Culpeper, J. (2011). 'It's not what you said, it's how you said it!": Prosody and impoliteness. En Linguistic Politeness Research Group (Eds.), Discursive approaches to politenss (pp. 57-83). Berlín: Mouton de Gruyter.

Fuentes, M. (2012). Análisis fonético-acústico de la conducta prosódica de los enunciados del tipo imperativo (petición y orden) del español de Santiago de Chile (Trabajo final de magíster no publicado). Santiago de Chile: Pontificia Universidad Católica de Chile.

Garrido, J. M. (2001). La estructura de las curvas melódicas del español: Propuesta de modelización. Lingüistica Española Actual, 23(2), 173-210.

Gil, J. (2007). Fonética para profesores de español: De la teoría a la práctica. Madrid: Arco Libros.

Hidalgo, A. (2002). Variación prosódica y diferencias de sexo. En torno a sus correlatos funcionales. Cuadernos de Filología. Estudios Lingüisticos, 7, 3-21.

Hidalgo, A. (2006a). Aspectos de la entonación española: viejos y nuevos enfoques. Madrid: Arco Libros.

Hidalgo, A. (2006b). La expresión de cortesía (atenuación) en español hablado: Marcas y recursos prosódicos para su reconocimiento en la conversación coloquial. En M. Villayandre Llamazares (Ed.), Actas del XXXV Simposio Internacional de la Sociedad Española de Lingüística (pp. 957-979). León: Universidad de Leon. Recuperado de http://www3.unileon.es/dp/dfh/SEL/actas.htm

Hidalgo, A. (2007). Sobre algunos recursos fónicos del español y su proyección sociopragmática: Atenuación y cortesía en la conversación coloquial. Quaderns de Filologia. Estudis Lingüistics, 12, 129-142.

Hidalgo, A. (2009). Modalización (des)cortés y prosodia: Estado de la cuestión en el ámbito hispánico. Boletín de Filología de la Universidad de Chile, 44(1), 161-195.

Hidalgo, A., y A. Cabedo (2011-2012). Observaciones sobre la importancia de la entonación en la enseñanza de E/LE: Aspectos metodológicos. Cauce, 34(35), 209-230.

Hidalgo, A., y Martínez, D. (2017). Hacia una propuesta metodológica para el estudio de la atenuación fónica en Es.Var.Atenuación. Soprag, Sociocultural Pragmatics. An International Journal of Spanish Linguistics, 5(1), 25-58.

Instituto Cervantes (2006). Plan curricular del Instituto Cervantes. Niveles de referencia para el español. Madrid: Instituto Cervantes. Recuperado de http://cvc.cervantes.es/ ensenanza/biblioteca_ele/plan_curricular/ 
Kawahara, H., Estill, J., y Fujimura, O. (2001). Aperiodicity extraction and control using mixed mode excitation and group delay manipulation for a high quality speech analysis, modification and synthesis system STRAIGHT. Second International Workshop on Models and Analysis of Vocal Emissions for Biomedical Applications, 2nd MAVEBA. Recuperado de http://www.isca-speech.org/archiv: 59-64.

Lahoz, J. (2007). La enseñanza de la entonación en el aula de ELE: Cómo, cuándo y por qué. Madrid: Universidad Complutense de Madrid.

Llisterri, J. (2001). Enseñanza de la pronunciación, corrección fonética y nuevas tecnologías. Recuperado de http://liceu.uab.es/_joaquim/publicacions/CorrFon_ NT_2001.pdf

Martínez, E., y Fernández, P. (2007). Manual de fonética española. Barcelona: Ariel.

Navarro Tomás, T. (1966). Manual de entonación española. Nueva York: Hispanic Institute.

Oplustil, P. (2016). Patrones melódicos de órdenes y peticiones a través de un análisis automático de la entonación. Estudios de Fonética experimental, 25, 233-261.

Orozco, L. (2005). Peticiones corteses y factores prosódicos. México: El Colegio de México.

Orozco, L. (2008). Peticiones corteses y factores prosódicos. En E. Herrera y P. Martín Butragueño (Eds.), Fonología instrumental. Patrones fónicos y variación (pp. 335-355). México: El Colegio de México.

Prieto, P. (Ed.) (2003). Teorías lingüisticas de la entonación. Barcelona: Ariel.

Roldán, Y. (2000). Correlatos acústicos de actos de habla atenuados del español de Chile. Onomázein, 5(10), 7-118. 